\title{
ESTUDIO DE DIVERSIDAD GENÉTICA DEL AGUACATE NATIVO EN NUEVO LEÓN, MÉXICO
}

\author{
STUDY OF GENETIC DIVERSITY OF NATIVE AVOCADO IN NUEVO LEÓN, MÉXICO
}

\author{
Adriana Gutiérrez-Díez ${ }^{1 *}$, Jesús Martínez-de la Cerda ${ }^{1}$, Eduardo A. García-Zambrano ${ }^{1}$, Leobardo \\ Iracheta-Donjuan $^{2}$, Jesús D. Ocampo-Morales ${ }^{1}$ e Ivón M. Cerda-Hurtado ${ }^{1}$
}

\begin{abstract}
${ }^{1}$ Facultad de Agronomía, Universidad Autónoma de Nuevo León. Francisco Villa s/n, Col. Ex-Hacienda El Canadá. 66054, Escobedo, N. L. México. Tel. y Fax (81) 13404399 ext. 3517. ${ }^{2}$ Campo Experimental Rosario Izapa, Instituto Nacional de Investigaciones Forestales, Agrícolas y Pecuarias. 30870, Tuxtla Chico, Chiapas, México.
\end{abstract}

*Autor para correspondencia (mcgudiez@aol.com)

\section{RESUMEN}

El estado de Nuevo León en México es centro de origen de Persea americana Mill. spp. drymifolia, como lo evidencian los vestigios de frutos encontrados en cuevas de la Sierra Madre Oriental. En este estado aún es posible encontrar plantas creciendo en hábitat silvestre y con características contrastantes a las de las variedades nativas que se cultivan en huertas familiares $y$ de traspatio. Esta diversidad representa una fuente valiosa de genes y combinaciones genéticas que puede utilizarse en programas de mejoramiento de Persea. Debido a que esta diversidad genética no se ha caracterizado, se planteó su estimación en $\mathbf{4 2}$ árboles de aguacate nativo con base en características de peso, longitud y diámetro de fruto, peso, longitud y diámetro de semilla y, longitud y diámetro de la cavidad de la semilla, además de las relaciones longitud/diámetro de fruto y peso de semilla/peso de fruto. De éstas, sólo los pesos de semilla y fruto, y las longitudes de cavidad de semilla y de fruto fueron clasificatorios para la especie. Los resultados hicieron evidente que la clasificación de la diversidad con estos caracteres morfológicos de frutos no quedó bien representada. Para evaluar de forma directa la diversidad genética, se generaron AFLP que permitieron diferenciar cinco variedades en el análisis de agrupamiento; el no agrupamiento de variedades denominadas con el mismo nombre local permitió detectar diferencias genéticas dentro de variedades de la raza mexicana. Cuando los datos moleculares se analizaron en forma conjunta con los datos morfológicos, se logró diferenciar once variedades, y se repitió el esquema de no agrupamiento de variedades con el mismo nombre local. La utilidad de los AFLP en el análisis de diversidad genética entre variedades de $P$. americana de la raza mexicana quedó evidenciada con estos resultados.

Palabras clave: Persea americana spp. drymifolia, diversidad genética, marcadores moleculares, AFLP.

\section{SUMMARY}

The State of Nuevo León in México is the center of origin of Persea americana Mill. spp. drymifolia, as evidenced by the remains of fruits found in caves of Nuevo Leon's Sierra Madre Oriental mountains. Within the state, native avocado plants have been found in the wild with different characteristics to the current varieties cultivated in family orchards. This diversity represents a valuable source of genes and genetic combinations that can be used in Persea breeding programs. Because this genetic diversity has not been studied, an analysis of 42 native avocado samples on the basis of the fruit traits: weight, length and diameter of fruit; weight, length and diameter of seed and length and diameter of the seed cavity; and relations between length/diameter of fruit and weight seed/weight fruit was carried out. Only seed weight, fruit weight, length of seed cavity and fruit length were useful for classification of the species. The results made clear that the classification based on morphological fruits traits did not represent the variability. Characterization by molecular markers offers the possibility to evaluate directly the genetic diversity. AFLP analysis allowed to distinguish five different varieties and the separation of varieties with the same local name, thus demonstrating genetic differences within varieties of the Mexican race.

When the molecular data were analyzed together with the morphological data, eleven varieties were differentiated and the separation of varieties with the same local name was possible again. The utility of AFLP in the analysis of genetic diversity between varieties of avocados of the Mexican race has been demonstrated with these results.

Index words: Persea americana spp. drymifolia, genetic diversity, molecular markers, AFLP.

\section{INTRODUCCIÓN}

México es considerado centro de origen de Persea americana Mill. En el caso de la raza mexicana o subespecie drymifolia los hallazgos de aguacates primitivos en áreas de la Sierra Madre Oriental del Estado de Nuevo León evidencian que el Estado es centro de origen de la misma (Sánchez-Pérez, 1999). En Nuevo León aún es posible encontrar plantas silvestres de $P$. americana que forman parte de la vegetación, cuyas características son contrastantes a las variedades mejoradas que se cultivan en diferentes sistemas agrícolas y de forma intensiva, tanto en huertas familiares como de traspatio. Estas formas de producción son importantes centros de experimentación, introducción de plantas y mejoramiento empírico, así como refugios de diversidad genética única que 
albergan genes que aún no han sido estudiados. Estos genes son de uso potencial en programas de mejoramiento genético de Persea. Las variedades "mejoradas" consisten de selecciones locales que se han cultivado por varios años y que los productores han seleccionando con base en su producción y calidad.

La raza mexicana de $P$. americana se caracteriza por su resistencia al frío, alto contenido de aceite y olor a anís de sus hojas en casi todos sus individuos; por lo general crece en altitudes mayores a $2000 \mathrm{~m}$. Sus hojas son de color verde oscuro pero los brotes jóvenes son verdes claros o rojizos; el fruto es de cáscara delgada, lisa y suave, la semilla puede estar adherida o suelta y sus cotiledones son lisos o ligeramente rugosos; es común que presenten fibra en la pulpa, aunque ésta no se encuentra en la mayoría de especies cultivadas (Barrientos-Priego y LópezLópez, 2002). Un problema importante de este género en Nuevo León, es la pérdida de esta diversidad debido a la práctica denominada "reposición de copas" mediante la cual las variedades menos aceptadas comercialmente, a juicio de los productores, son sustituidas por variedades "mejoradas" de mayor rendimiento y calidad (sabor, peso y tamaño de fruto).

El principal problema para identificar la variabilidad genética del aguacate nativo en Nuevo León es la diversidad de nombres locales con que se denominan a las variedades. La identificación tradicional de las plantas se hace por caracterización fenotípica, procedimiento lento y limitado porque la expresión de los caracteres cuantitativos está sujeta a fuerte influencia ambiental. Los marcadores moleculares permiten identificar, clasificar y aprovechar la diversidad genética existente en los genomas de las plantas, ya que las diferencias o similitudes a nivel del ADN entre individuos se observan en forma directa.

La generación de marcadores moleculares en aguacate ha sido lenta con respecto a otras especies. Varios estudios se han enfocado a desarrollar marcadores en este cultivo: isoenzimas (Torres y Bergh, 1998), RFLP (Davis et $a l .$, 1998), SSR (Ashworth y Clegg, 2003; Ashworth et al., 2004), VNTR (Mhameed et al., 1996) y RAPD (Fiedler et al., 1998; Sharon et al., 1998; Kobayashi et al., 2000); algunos se han enfocado a caracterizar la diversidad genética (Davis et al., 1998; Fiedler et al., 1998; Gallo-Llobet, 1999; Ashworth y Clegg, 2003; Schnell et al., 2003).

Los AFLP (Amplified Fragment Length Polymorphism) o polimorfismos en la longitud de los fragmentos amplificados, son marcadores moleculares de alta eficiencia desarrollados por Vos et al. (1995) como una técnica para generar huellas genéticas. Se han utilizado amplia- mente en estudios de diversidad genética e identificación de variedades de diversos frutales: Mangifera indica L. (Lavi et al., 1998), Vitis vinifera (Riaz et al., 2007), Pyrus spp. (Monte-Corvo et al., 2000; Yamamoto et al., 2002) y Carica papaya (Mishra et al., 2007), por citar algunos. En aguacate los AFLP se han utilizado para estudiar la diversidad genética de bancos o colecciones de germoplasma (Gallo-Llobet, 1999). Virk et al. (1998) estimaron la diversidad genética de 18 accesiones, mientras que Rodríguez et al. (2003) identificaron 17 genotipos de la colección de germoplasma del IIFT en Cuba. Chao et al. (2003) estudiaron las relaciones genéticas entre accesiones de California y México, mientras que BarrientosPriego y colaboradores (Com. personal) ${ }^{1}$ utilizaron AFLP para el análisis genético de aguacate mexicano.

La clasificación y conservación del germoplasma vegetal debe verse como una actividad que permite preservar este patrimonio nacional de biodiversidad, lo cual requiere de un esfuerzo importante para conservar los genotipos de plantas cultivadas y silvestres que actualmente son amenazadas al ser sustituidas por variedades mejoradas. El objetivo de este trabajo fue estimar la diversidad genética de $P$. americana Mill. spp. drymifolia en los municipios de Aramberri y Zaragoza del Estado de Nuevo León, mediante caracteres morfológicos de fruto y marcadores moleculares tipo AFLP.

\section{MATERIALES Y MÉTODOS}

Se colectaron cinco frutos maduros y cinco hojas jóvenes ubicadas en de las puntas de las ramas de 42 árboles de aguacate en los municipios de Aramberri y Zaragoza, N. L., de huertas familiares, traspatio, y en su hábitat silvestre; cada árbol correspondió a una muestra de las diferentes variedades. La identificación de los árboles se hizo de acuerdo con el nombre local proporcionado por los productores o habitantes de la región; a las muestras de las que se desconocía el nombre local se denominaron 'Criollos'; las muestras colectadas en hábitat silvestre se denominaron 'Silvestres'; se denominó muestras mejoradas a las injertadas sobre algún patrón de aguacate nativo; las muestras nativas corresponden a las propagadas por semillas provenientes de la región (Cuadro 1).

En los frutos maduros (listos para consumo) se evaluaron los siguientes caracteres morfológicos, de acuerdo con los descriptores morfológicos para fruto de aguacate del International Plant Genetic Resources Institute (IPGRI, 1995): peso (gramos), longitud y diámetro (centímetros), peso de semilla (gramos), longitud y diámetro de

\footnotetext{
${ }^{1}$ Dr. Alejandro F. Barrientos-Priego. Especialista en aguacate. Dpto. Fitotecnia, Universidad Autónoma Chapingo.
} 
semilla (centímetros), longitud y diámetro de la cavidad de la semilla (centímetros), relación longitud/diámetro de fruto, y relación peso de semilla/peso de fruto. Debido a que no existen estudios previos sobre el tema, para determinar las variables de mayor peso en la caracterización y para estimar y describir el comportamiento de las variedades con respecto a cada característica, se estimó la media, desviación estándar y el coeficiente de variación de cada una.

La extracción del ADN foliar se hizo por el método del ruptor celular con el kit FastDNA ${ }^{\circledR}$ (Qbiogene, Inc., CA). La cuantificación del ADN se hizo mediante fluorimetría; la calidad del ADN se determinó en gel de agarosa $1 \%$. La generación de AFLP se llevó a cabo con el estuche comercial IRDye ${ }^{\mathrm{TM}}$ Fluorescent AFLP Kit for Large Plant Genome Analysis de LI-COR ${ }^{\circledR}$ Biosciencies (Lincoln, NE, EE. UU.). Las etapas de digestión del ADN y ligación de adaptadores se hicieron de acuerdo con el protocolo incluido en el estuche comercial. La etapa de preamplificación se modificó al ajustar el volumen de reacción a $27 \mu \mathrm{L}$; la mezcla consistió de ADN digerido y ligado $(2.5 \mu \mathrm{L})$, mezcla de preamplificación AFLP (20 $\mu \mathrm{L})$, solución amortiguadora $10 \mathrm{X}(0.9 \mathrm{X}), \mathrm{MgCl}_{2}(1.5$ $\mathrm{mM}$ ) y Taq ADN polimerasa (2.5 U). El programa térmico de incubación fue 20 ciclos a $94{ }^{\circ} \mathrm{C}$ por $30 \mathrm{~s}, 1 \mathrm{~min}$ a $56{ }^{\circ} \mathrm{C}$ y $1 \mathrm{~min}$ a $72{ }^{\circ} \mathrm{C}$. El producto de preamplificación se diluyó a una concentración de 1:40.

Para seleccionar los iniciadores se probaron las 64 combinaciones propuestas en el estuche comercial, y se seleccionaron las 15 que proporcionaron mayor definición de bandas. En la amplificación selectiva se manejaron reacciones dúplex para los iniciadores $E c o$ R1-700 IRDye ${ }^{\mathrm{TM}}$ y EcoR1-800 IRDye ${ }^{\mathrm{TM}}$ conforme al protocolo del estuche comercial. El programa térmico fue el propuesto en el mismo protocolo. La separación de los productos de amplificación se hizo por electroforesis en gel de poliacrilamida $6.5 \%$ en un secuenciador LI-COR ${ }^{\circledR} 4200$ (Lincoln, NE, EE. UU.). Los parámetros de colecta fueron de 16 "bits", $1500 \mathrm{~V}, 40 \mathrm{~W}, 40 \mathrm{~mA}$ y $45^{\circ} \mathrm{C}$; la velocidad del escáner fue de 4 . El análisis de identificación, presencias y ausencias de bandas se hizo con el programa SAGA ${ }^{\mathrm{MX}}$ (LI-COR, Lincoln, NE, EE. UU.). En el análisis molecular se supuso que las bandas con el mismo peso molecular en muestras diferentes son idénticas; y a cada muestra se asignó el número $1 \mathrm{a}$ la banda presente y el número 0 a la ausencia de banda, para formar la matriz de datos binarios requerida para determinar los índices de similitud con el coeficiente de Gower (1-S) (Franco e Hidalgo, 2003).

El análisis de datos morfológicos de los frutos fue mediante componentes principales y con base en éste se aplicó el análisis de agrupamiento con el método UPGMA (Unweighted Pair Groups Method with Arithmetic Averages) con distancia Gower (1-S). Los análisis estadísticos se llevaron a cabo con el programa InfoStat/P versión 2006p.3 (InfoStat, 2006). El análisis de agrupamiento de los datos moleculares fue con variables estandarizadas. Con los datos morfológicos de frutos y los datos binarios se construyó una matriz de datos mixtos que sirvió para hacer el análisis de agrupamiento comparativo entre los grupos formados con el método de UPGMA con distancia Gower (1-S). El programa estadístico utilizado en el análisis de los datos moleculares y de datos mixtos fue InfoGen/P versión 2006p.1 (Balzarini y Di Rienzo, 2003). En todos los casos se calculó el coeficiente de correlación cofenética.

\begin{tabular}{|c|c|c|c|}
\hline Nombre local & ID & Estado de la muestra & Fuente de colecta \\
\hline 'Huevo de paloma' & P1 & Mejorada & $\mathrm{HF}$ \\
\hline ‘Campeón’’ & P2, P6 & Mejorada & HF \\
\hline 'Pagua' & P3, P24 & Mejorada & $\mathrm{HF}$ \\
\hline 'Plátano grueso' & $\mathrm{P} 4, \mathrm{P} 26$ & Mejorada & $\mathrm{HF}$ \\
\hline 'Criollo' & P5, P16, P18, P44 & Nativa & $\mathrm{HF}$ \\
\hline 'Plátano delgado' & $\mathrm{P} 7, \mathrm{P} 11, \mathrm{P} 12, \mathrm{P} 13, \mathrm{P} 14, \mathrm{P} 15, \mathrm{P} 21$ & Mejorada & $\mathrm{HF}$ \\
\hline 'Verde' & P9 & Mejorada & $\mathrm{HF}$ \\
\hline 'María Elena' & P19, P33 & Mejorada & HF \\
\hline ‘Plátano’' & $\mathrm{P} 20, \mathrm{P} 27, \mathrm{P} 28, \mathrm{P} 29, \mathrm{P} 30, \mathrm{P} 38$ & Mejorada & $\mathrm{HF}$ \\
\hline ‘Calabo' & $\mathrm{P} 23$ & Mejorada & HF \\
\hline 'Leonor' & $\mathrm{P} 25$ & Mejorada & $\mathrm{HF}$ \\
\hline ‘Chino’' & $\mathrm{P} 32$ & Mejorada & $\mathrm{HF}$ \\
\hline 'Criollito' & P39 & Nativa & HF \\
\hline 'Silvestre' & P45, P46, P47, P48, P49, P50, P53, P56, P57 & Silvestre & HS \\
\hline ‘Criollo’ & P59, P60 & Mejorada & HT \\
\hline
\end{tabular}

$\mathrm{HF}=$ Huerta familiar; HS = Hábitat silvestre; HT = Huerta de traspatio. 


\section{RESULTADOS Y DISCUSIÓN}

De los 42 árboles colectados se identificaron 11 nombres locales diferentes a los denominados 'Criollos', 'Criollitos' y 'Silvestres' (Cuadro 1). En el caso de las muestras identificadas como variedad 'Plátano' (P20, P27, P28, P29, P30 y P38) no se pudo definir si pertenecen a la variedad 'Plátano delgado' o 'Plátano grueso'.

Las variables con coeficientes de variación mayores a $20 \%$ se consideraron clasificatorias para la especie y fueron: peso de semilla (39.42 \%), peso de fruto (38.42\%), longitud de la cavidad de semilla (24.82\%) y longitud de fruto $(20.34 \%)$. Valores mayores a $50 \%$ sugieren que las variables tienen alta variabilidad dentro de una especie determinada y los valores menores a $20 \%$ indican poca variabilidad de la especie (Franco e Hidalgo, 2003). En peso de semilla y peso de fruto las medias más altas fueron de $69.76 \mathrm{~g}$ y $251.40 \mathrm{~g}$, respectivamente, correspondientes a P26 ('Plátano grueso'); los valores más bajos para estas variables fueron de 7.08 y $10.53 \mathrm{~g}$, respectivamente, para P57 ('Silvestre'). En la longitud de la cavidad de semilla, la media más alta correspondió a P21 ('Plátano delgado') con $11.46 \mathrm{~cm}$, y la más baja correspondió a P57 con $2.60 \mathrm{~cm}$. Para longitud de fruto el valor más alto fue $12.76 \mathrm{~cm}$ y correspondió a P4 ('Plátano grueso') mientras que el valor más bajo fue de $2.88 \mathrm{~cm}$ para P57. Como se observa, los valores más bajos para las variables clasificatorias correspondieron a P57, mues- tra que fue colectada en hábitat silvestre y presentó características de fruto muy contrastantes al resto de las muestras colectadas (Figura 1). De igual modo, en las variables no clasificatorias las medias más bajas correspondieron a P57.

El análisis de componentes principales $(\mathrm{CP})$ permitió determinar la relación entre las variables y la semejanza de las muestras. La Figura 2 muestra que los CP1 y CP2 representaron $75.8 \%$ de la varianza absoluta; valores mayores al rango de 60-70 \% explican un porcentaje razonable de la variabilidad total de las muestras (Balzarini et al., 2006). En el CP1 (59.7 \% de la varianza), las variables con mayor influencia en la caracterización de las muestras fueron: peso de fruto, peso de semilla y longitud de fruto; en el CP2 (16.1\% de la varianza), las variables diámetro de semilla y longitud de la cavidad de semilla fueron las de mayor influencia. Esto concuerda con los resultados del análisis de los coeficientes de variación. La correlación cofenética del análisis de componentes principales fue de 0.984. En el análisis de CP se formaron seis grupos, en donde P57 fue la más distante. Las muestras P45 y P48 que corresponden a criollos colectados en hábitat silvestre formaron un grupo, mientras que P53 ('Silvestre'), P56 ('Silvestre') y P11 ('Plátano delgado') forman otro grupo. P25 identificada como 'Leonor' quedó separada del resto de las muestras, al igual que P21 identificada como 'Plátano delgado'. El resto de las variedades se agruparon.

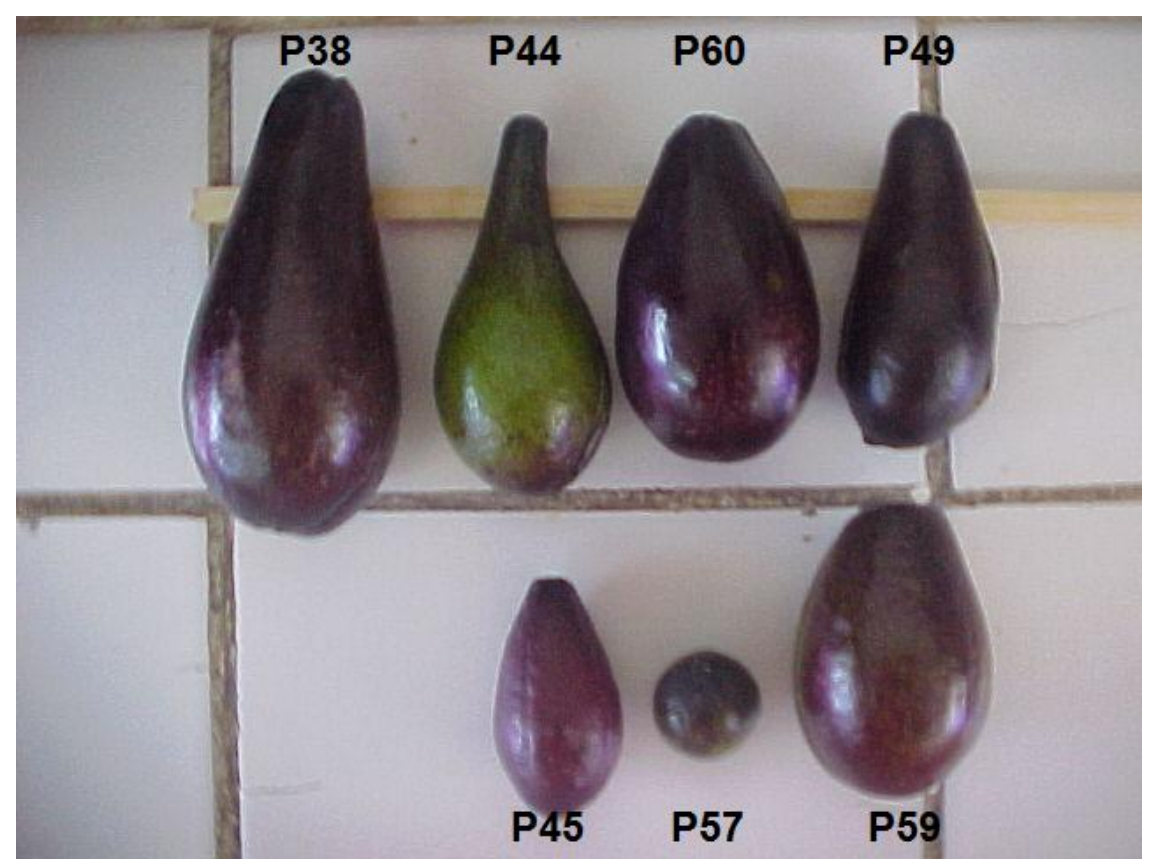

Figura 1. Comparación de tamaño y formas de frutos de Persea americana Mill. provenientes de Aramberri y Zaragoza en el Estado de Nuevo León, México. La identificación de las muestras es la indicada en el Cuadro 1. 


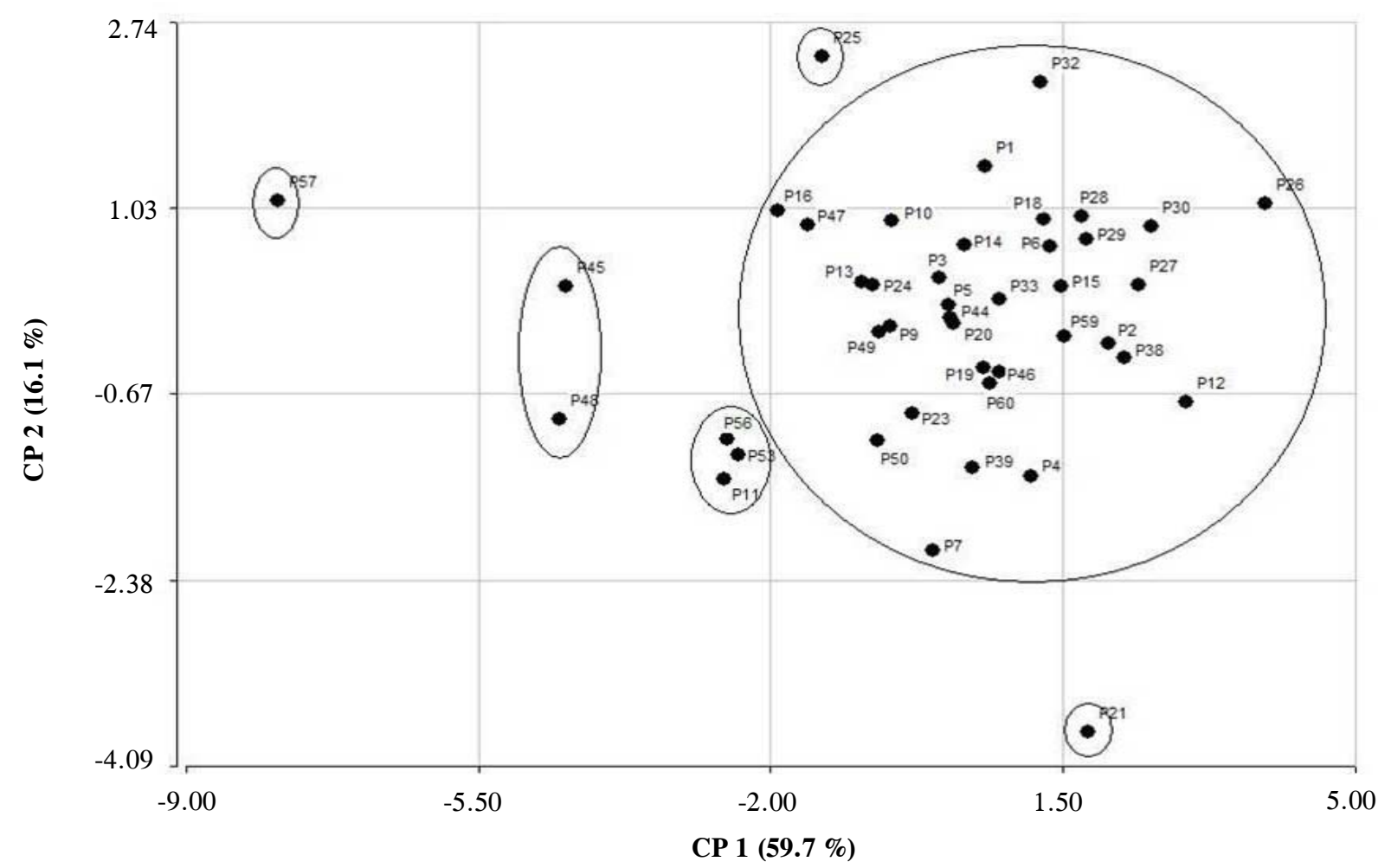

Figura 2. Ordenamiento producido por las variables: peso de semilla, peso de fruto, longitud de la cavidad de la semilla y longitud de fruto en 42 árboles de Persea americana Mill. provenientes de los municipios de Aramberri y Zaragoza en el Estado de Nuevo León, México.

El análisis de agrupamiento se efectuó con las variables peso de semilla, peso de fruto, longitud de la cavidad de semilla y longitud de fruto, porque mostraron la mayor influencia en la clasificación de las muestras; se incluyeron, además, la relación longitud/diámetro de fruto y la relación peso de semilla/peso de fruto, porque incrementaron el valor de la correlación cofenética (0.888). En el dendograma (Figura 3) se muestra que a una distancia de corte de 0.23 se forman cuatro grupos, de los cuales dos coinciden con los formados por el análisis de componentes principales: Grupo I (P57) y Grupo II (P21, 'Plátano delgado'). En el grupo IV quedaron agrupadas cinco muestras identificadas como 'Silvestres' (P45, P47, P48, P53 y P56), un 'Criollo' (P16) y las muestras 'Huevo de paloma' (P1), 'Leonor' (P25) y 'Chino' (P32). El resto de las muestras formaron el Grupo III, en que todas las muestras identificadas como 'Plátanos', 'Plátano grueso' y 'Plátano delgado', quedaron incluidas, con excepción de la muestra P21.

Aun cuando las diferencias fenotípicas entre frutos de diferentes variedades fueron claras (Figura 1), sólo fue posible la identificación de P57. Las distancias de eslabonamiento dentro del dendograma reflejan las diferencias e inconsistencia entre las variedades presumiblemente iguales. Se esperaba que las muestras identificadas como de la misma variedad se agruparan, lo que no fue evidente; esto concuerda con Gama-Campillo y Gómez-Pompa (1992), quienes aseguran que el tamaño del fruto es una característica que no ayuda a diferenciar entre formas silvestres y cultivadas de plantas de aguacate, ya que los árboles producen frutos de diferentes tamaños. Rodríguez et al. (2003), en su estudio sobre análisis de grupos ecológicos de aguacate, aseguran que éstos no quedan bien representados cuando la clasificación se hace con caracteres morfológicos de fruto. Por ser el aguacate una especie de polinización abierta, la diversidad genética es amplia y al utilizar frutos en vez de partes vegetativas existe la probabilidad de que se evalúe la combinación genética de dos plantas diferentes que pueden o no ser de la misma variedad; por tanto, es necesario evaluar otros caracteres que reflejen en forma directa la información genética de las plantas, como patrón de floración, longitud, ancho, forma, y borde de hojas.

Para el análisis de datos moleculares se seleccionaron 683 marcadores AFLP producidos por 15 combinaciones de iniciadores: M-CAA_E-AAG, M-CAA_E-ACT, MCAC_E-AGC, M-CAG_E-ACG, M-CTA_E-AGC, MCTA_E-AGG, M-CTC_E-AAG, M-CTC_E-ACA, MCTC_E-ACT, M-CTC_E-AGC, M-CTG_E-AGC; MCTT_E-AAC, M-CTT_E-ACA, M-CTT_E-ACT y MCTT_E-AGC. Los productos de amplificación seleccionados estuvieron en el rango de $50 \mathrm{a} 400 \mathrm{pb}$; ninguna banda 
fue monomórfica. En la Figura 4 se muestran los marcadores AFLP de la combinación de iniciadores M-CTC_EACT. El análisis de agrupamiento de los datos moleculares (Figura 5) definió (distancia de 0.14) dos grupos y ocho muestras sin agrupar: P9 ('Verde'), P44 ('Criollo'), P38 ('Plátano'), P4 ('Plátano grueso'), P3 ('Pagua'), P29 ('Plátano'), P39 ('Criollito') y P23 ('Calabo'). En este caso, el no agrupamiento o separación de P9, P44, P3, P39 y P23 ('Verde', 'Criollo', 'Pagua', 'Criollito' y 'Calabo', respectivamente) demuestra que las variedades son diferentes; sin embargo, el no agrupamiento de P38, P4 y P29 ('Plátanos', 'Plátano grueso' y 'Plátano', respectivamente) deja a discusión si las muestras se identificaron erróneamente por parte de los productores, o si existe diversidad genética dentro de variedades, y en este último caso será necesario usar otro tipo de marcadores moleculares como SSR (Ashworth et al., 2007).
El Grupo I está formado por P48, P46 y P45, identificadas como muestras 'Silvestres'. El Grupo II quedó conformado por 32 muestras colectadas en las tres fuentes definidas en el Cuadro 1 (huertas familiares, huertas traspatio y hábitat silvestre); al disminuir la distancia de corte (0.09), las muestras identificadas como 'Plátano' (P30, $\mathrm{P} 15, \mathrm{P} 13, \mathrm{P} 12)$ y 'Plátano delgado' (P11), se agruparon; mientras que P56 ('Silvestre'), P50 ('Silvestre') y P32 ('Chino') se separaron. El agrupamiento a distancias muy cortas (0.015) se dio entre P6 ('Campeón'), P7 ('Plátano delgado') y P1 ('Huevo de paloma') y entre P53 ('Silvestre') y P33 ('María Elena') lo que demuestra que están estrechamente relacionadas. Los agrupamientos entre 'Plátanos' y otras variedades ocurrieron a distancias mayores y generaron ambigüedad acerca de la conformación genética de las variedades de las huertas familiares, principalmente de las variedades "mejoradas" o injertadas.

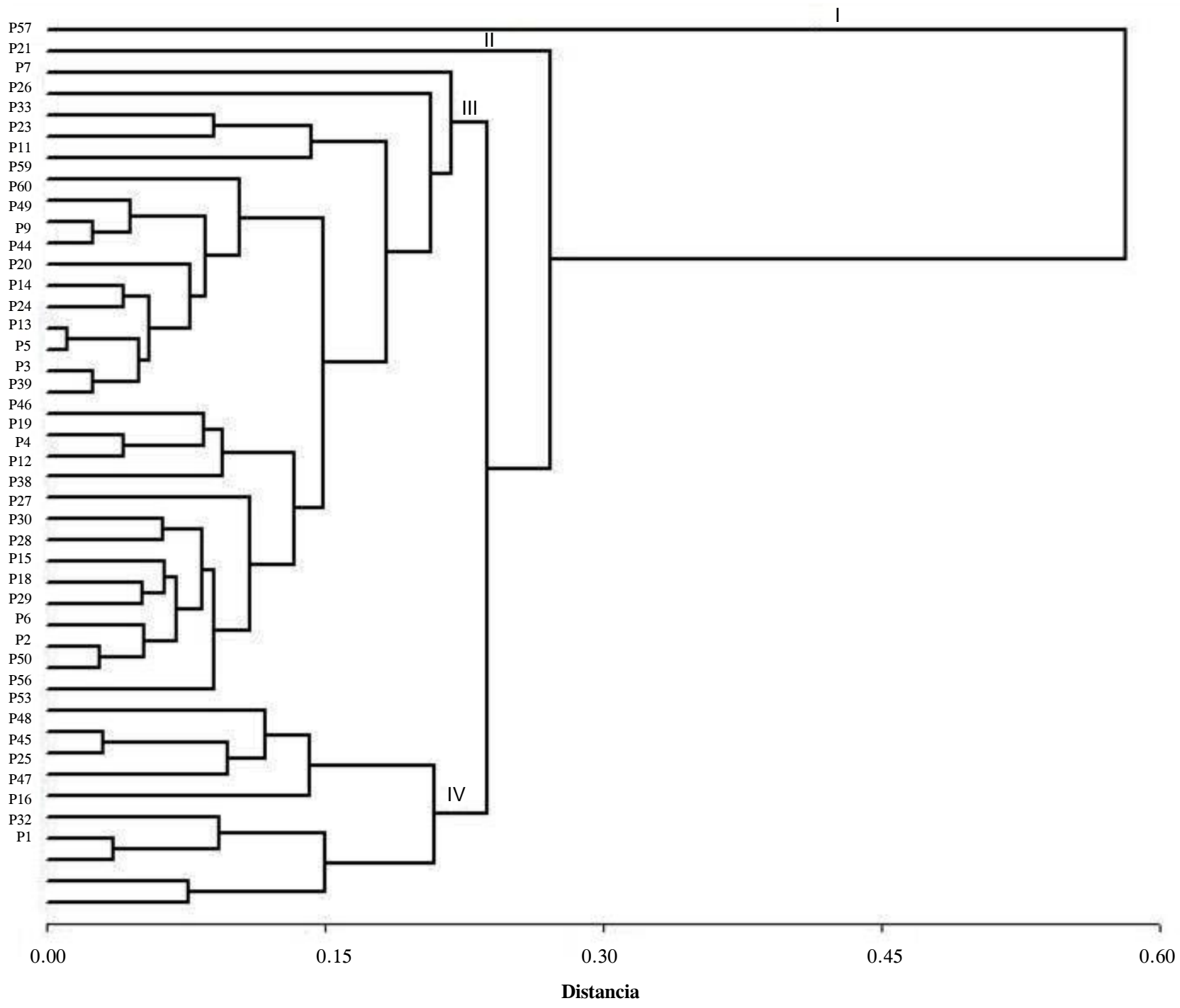

Figura 3. Dendograma de datos morfológicos de frutos de 42 árboles de Persea americana Mill. provenientes del Estado de Nuevo León, obtenido por UPGMA y distancia Gower (1-S) con coeficiente de correlación cofenética de $\mathbf{0 . 8 8 8}$. 


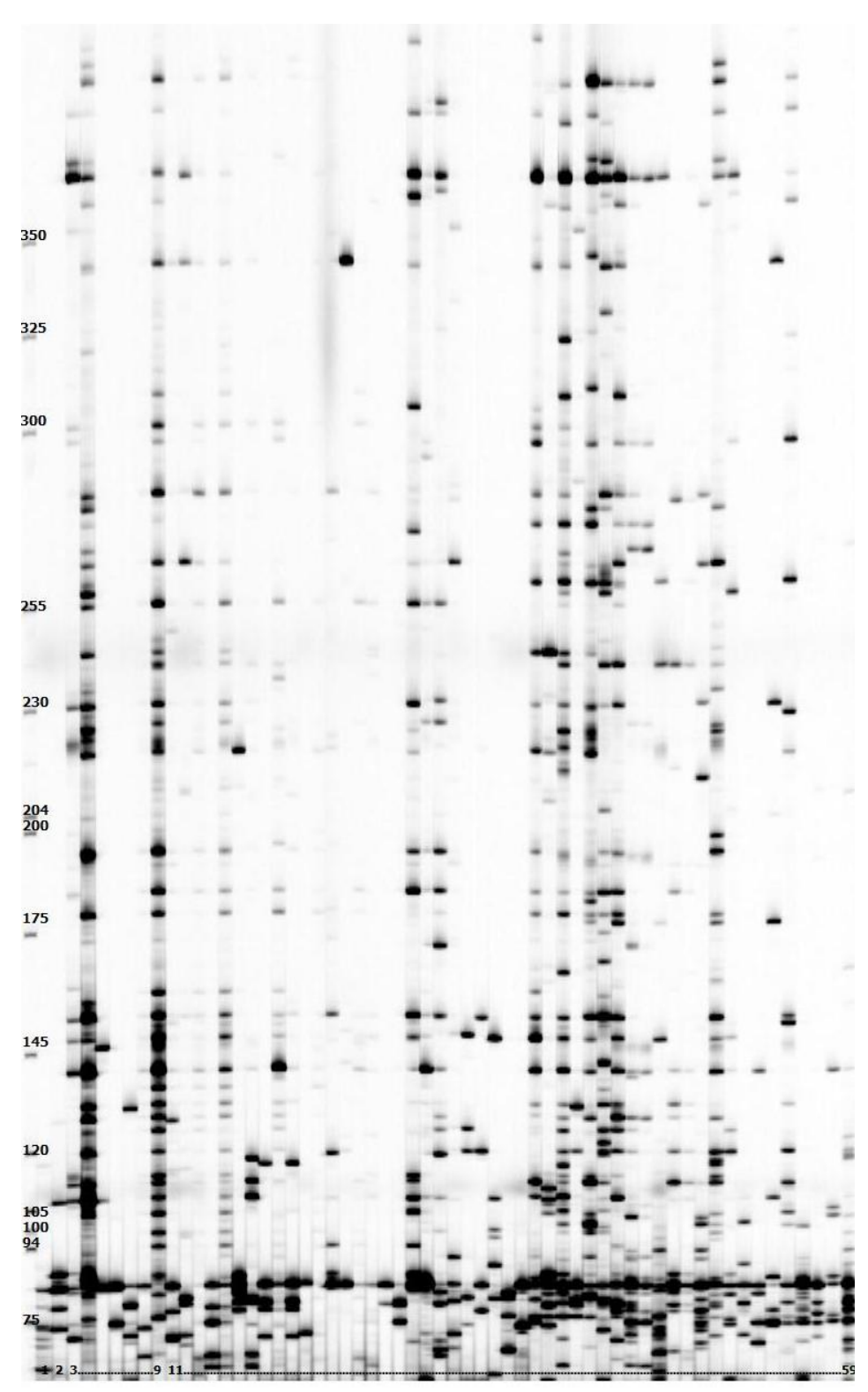

Figura 4. Marcadores AFLP producidos por los iniciadores M-CTC_E-ACT, en Persea americana Mill. provenientes de los municipios de Aramberri y Zaragoza en el Estado de Nuevo León, México.

En la Figura 6 se muestra el dendograma que corresponde al análisis conjunto de los datos moleculares y fenotípicos, o datos mixtos; a una distancia de corte de 0.13 se definió el Grupo I formado por 31 muestras, mientras que 11 permanecieron sin agrupar: P48 ('Silvestre'), P57 ('Silvestre'), P45 ('Silvestre'), P9 ('Verde'), P44 ('Criollo'), P4 ('Plátano grueso'), P3 ('Pagua'), P56 ('Silvestre'), P50 ('Silvestre'), P38 ('Plátano') y P29 ('Plátano'). La separación de P9, P44, P4 y P3 coincidió con lo obtenido en el análisis de datos moleculares; sin embargo se repite el esquema de separación de las muestras P4 y P38 y P29 identificadas como 'Plátanos'; esto evidenció la diversidad genética dentro de esta variedad. La separación de P48, P57, P45, P56 y P50 identificadas como 'Silves- tres', es una diferencia del análisis de datos mixtos con respecto al análisis de los datos moleculares.

$\mathrm{Al}$ aumentar la distancia de corte del gráfico a 0.21 , P57 y P45 ('Silvestres') se agruparon; estas muestras se caracterizan por presentar los menores valores de longitud de fruto y longitud de semilla. Otro agrupamiento fue el formado por P56 y P50 ('Silvestres'). Al ir aumentando la distancia de corte fue evidente la influencia de las variables fenotípicas en la conformación de los grupos; así, el grupo formado por P48, P57 y P45 se caracterizó por poseer los valores más bajos de peso de semilla. Dentro del Grupo I se encontraron variedades nativas, de hábitat silvestre y "mejoradas". Al analizar en forma detallada el 
esquema de agrupamiento, se observó que P26 y P27 se agruparon a menor distancia; una característica importante de estas muestras es que fueron las que presentaron el mayor peso de fruto y están identificadas como 'Plátano grueso' y 'Plátano' respectivamente, por lo que se puede concluir que P27 pertenece a la variedad 'Plátano grueso'.

Hasta ahora la utilización de AFLP en estudios de diversidad genética de aguacate es reciente, y en todos los casos se ha logrado diferenciar accesiones de bancos de germoplasma de acuerdo con sus tres grupos o razas de origen, pero no existen estudios sobre la diversidad genética dentro de razas. Los resultados demuestran que la utilización de AFLP permite estimar la diversidad genética intervarietal de la raza mexicana de $P$. americana cuando los datos moleculares se combinan con los datos morfológicos. Cuando solamente se utilizan datos moleculares generados por los AFLP, la diferenciación entre variedades fue ambigua, por lo que es necesario utilizar marcadores moleculares más específicos como los SSR. Chao et al. (2003) demostraron la eficacia y la facilidad de los marcadores AFLP para caracterizar accesiones y estudiar germoplasma en aguacate con base en las razas de origen; sin embargo, no se ha documentado su utilidad en la diferenciación intravarietal.

La utilidad de los AFLP en la estimación de la diversidad de aguacates mexicanos consistirá en la detección de una banda o bandas únicas representativas de cada variedad; una oportunidad para su utilización se abre con la identificación de bandas relacionadas con caracteres muy específicos de los mismos. Este es el primero de una serie de trabajos que se están realizando en Persea en el Estado de Nuevo León, para destacar la importancia del recurso genético del aguacate nativo, que en un futuro servirá para generar acciones de conservación y de mejoramiento genético del género Persea.

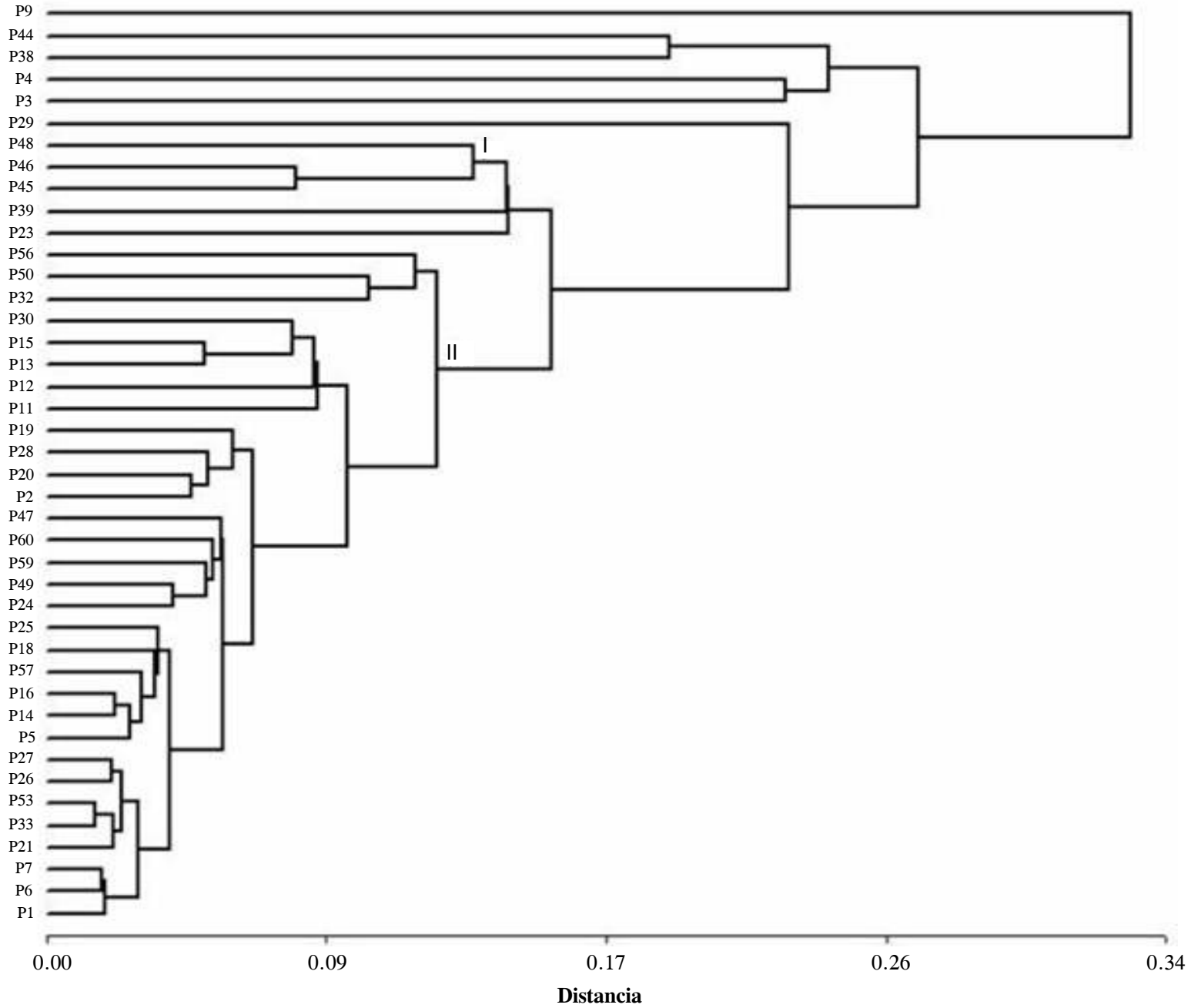

Figura 5. Dendograma de datos moleculares de AFLP de 42 árboles de Persea americana Mill. provenientes del Estado de Nuevo León obtenido por UPGMA y distancia Gower (1-S) con coeficiente de correlación cofenética de 0.990 . 


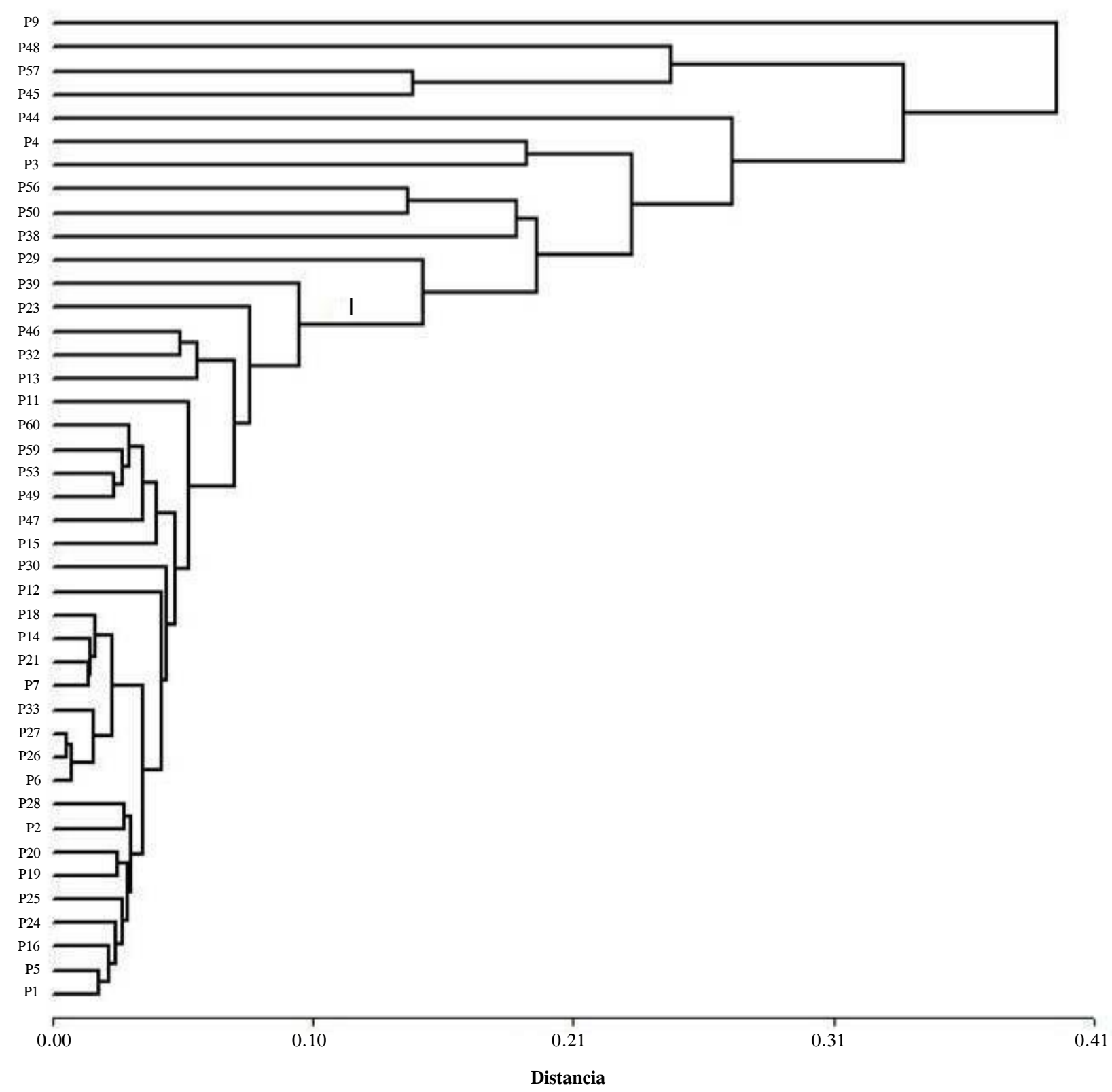

Figura 6. Dendograma de datos moleculares y fenotípicos de 42 árboles Persea americana Mill. provenientes del Estado de Nuevo León obtenido por UPGMA y distancia Gower (1-S) con coeficiente de correlación cofenética de 0.97.

\section{CONCLUSIONES}

La incorporación de las relaciones: longitud de fruto/diámetro de fruto, y peso de semilla/peso de fruto en el análisis de agrupamiento, incrementaron la información de similitud entre las muestras, ya que evaluaron con mayor exactitud la influencia del fruto y de la semilla en la formación de los grupos. El patrón de agrupamiento producido por la caracterización molecular de las muestras es diferente al patrón de caracterización morfológica de fruto. La separación mediante marcadores moleculares de las variedades: 'Verde' (P9), 'Criollo' (P44), 'Pagua' (P3),
'Criollito' (P39) y 'Calabo' (P23) corrobora la existencia de diversidad genética entre variedades. Contrario a lo esperado, el análisis de agrupamiento de los datos moleculares permitió definir solamente un grupo coincidente con el análisis de datos morfológicos. El análisis de agrupamiento conjunto de datos morfológicos y moleculares permitió separar 11 muestras y definir grupos. La separación de las mismas variedades identificadas con el mismo nombre local en los análisis de datos moleculares, y de datos conjuntos morfológicos y moleculares, permitió evidenciar divisiones genéticas dentro de variedades de aguacate de la raza mexicana. 


\section{AGRADECIMIENTOS}

A la Secretaría de Educación Pública que a través de su Programa de Mejoramiento del Profesorado (PROMEP) proporcionó el financiamiento del proyecto del que se derivó esta investigación. De igual forma, un sincero agradecimiento a los productores de aguacate del municipio de Aramberri, N. L., que colaboraron incondicionalmente y quienes están muy interesados en la difusión, defensa y estudio del aguacate nativo del Estado de Nuevo León.

\section{BIBLIOGRAFÍA}

Ashworth V E T M, M T Clegg (2003) Microsatellite markers in avocado (Persea americana Mill.): genealogical relationships among cultivated avocado genotypes. J. Heredity 94:407-415.

Ashworth V E T M, M C Kobayashi, M de la Cruz, M T Clegg (2004) Microsatellite markers in avocado (Persea americana Mill.): development of dinucleotide and trinucleotide markers. Sci. Hort. 101:255-267.

Ashworth V E T M, H Chen, M T Clegg (2007) Avocado. In: Genome Mapping and Molecular Breeding in Plants, Vol. 4. Fruits and Nuts. C Kole (ed). Springer-Verlag. Berlin Heidelberg. pp:325-329

Balzarini M, J Di Renzo (2003) Info-Gen: Software para Análisis Estadístico de Datos Genéticos. Facultad de Ciencias Agropecuarias. Universidad Nacional de Córdoba. Argentina.

Balzarini M, A Arroyo, C Bruno, J Di Renzo (2006) Análisis de Datos de Marcadores con Info-Gen. In: XXXV Congreso Argentino de Genética, San Luis, Argentina. 91 p.

Barrientos-Priego A, L López-López (2002) Historia y genética del aguacate. In: Memoria de la Fundación Salvador Sánchez Colín. Centro de Investigaciones Científicas y Tecnológicas del Aguacate en el Estado de México. Coatepec de Harinas, México. pp:100-121.

Chao C T, A F Barrientos-Priego, J C Reyes-Alemán, P S Devanand (2003) Relaciones genéticas entre accesiones de aguacate de California y de México, caracterizadas por marcadores AFLP. In: Proc. V World Avocado Congress. Research Conference. Granada-Málaga, Spain. 19-24 october. 1:208-209.

Davis J, D Henderson, M Kobayashi, M T Clegg, M T Clegg (1998) Genealogical relationships among cultivated avocado as revealed trough RFLP analyses. J. Heredity 89:319:323.

Fiedler J, G Bufler, F Bangerth (1998) Genetic relationships of avocado (Persea americana Mill.) using RAPD markers. Euphytica 101:249-255.

Franco T L, R Hidalgo (eds) (2003) Análisis Estadístico de Datos de Caracterización Morfológica de Recursos Fitogenéticos. Boletín Técnico No. 8. Instituto Internacional de Recursos Fitogenéticos. Cali, Colombia. 89 p.

Gallo-Llobet L (1999) Sustainable agriculture: the role of integrated management of root rot (Phtytophtora cinnamomi Rands) in avocado (Persea americana Mill.). In: Summary Reports of European Commission supported STD-3 projects (19921995). Technical Centre for Agricultural and Rural Cooperation.

Gama-Campillo L, A Gómez-Pompa (1992) An ethnoecological approach for the study of Persea: a case study in the maya area.
In: Proc. of II World Avocado Congress. Opening Session Conference. California, USA 1991. 1:11-17.

InfoStat (2006) InfoStat versión 2006. Grupo InfoStat. Facultad de Ciencias Agropecuarias. Universidad Nacional de Córdoba. Argentina. $314 \mathrm{p}$

IPGRI (1995) Descriptors for Avocado (Persea spp.). Internacional Plant Genetic Resources Institute. Rome, Italy. $52 \mathrm{p}$

Kobayashi M, J-Z Lin, J Davis, L Francis, M T Clegg (2000) Quantitative analysis of avocado outcrossing and yield in California using RAPD markers. Sci. Hort. 86:135-149.

Lavi U, D Sharon, S Mhameed, H Kashkush, A Adato, D Kaufman, P B Cregan, E Lahav, E Tomer, J Hillel (1998) Molecular markers in tropical and subtropical horticulture. Acta Hort. (ISHS). 461:49-54 (http://www.actahort.org/books/461/ 461_3.htm).

Mhameed S D, D Sharon, J Hillel, E Lahav, D Kaufman, U Lavi (1996) Level of heterozygosity and mode of inheritance of variable number of tandem repeat loci in avocado. J. Amer. Soc. Hort. Sci. 121:778-782.

Mishra M, R Chandra, S Saxena (2007) Papaya. In: Genome Mapping and Molecular Breeding in Plants. Vol. 4. Fruits and Nuts. C Kole (ed). Springer-Verlag. Berlin Heidelberg. pp:343-351.

Monte-Corvo L, L Cabrita, C M Oliveira, J M Leitao (2000) Assessment of genetic relationships among Pyrus species and cultivars using AFLP and RAPD markers. Gen. Resources Crop Evol. 47:257-265.

Riaz S, A Doligez, R J Henry, M A Walker (2007) Grape. In: Genome Mapping and Molecular Breeding in Plants. Vol.4. Fruits and Nuts. C. Kole (ed). Springer-Verlag. Berlin Heidelberg. pp:63-101.

Rodríguez N N, W Rhode, C González-Arencibia, I M RamírezPérez, J L Fuentes-Lorenzo, M A Román-Gutiérrez, X Xiqués-Martín, D Becker, J B Velásquez-Palenzuela (2003) Caracterización morfológica, bioquímica y molecular de cultivares de aguacatero (Persea americana Mill.) en $\mathrm{Cu}-$ ba. In: Proc. V World Avocado Congreso. Research Conference. Granada-Málaga, Spain. 19-24 october. 1:47-53.

Sánchez-Pérez J (1999) Recursos genéticos de aguacate (Persea americana Mill.) y especies afines en México. Rev. Chapingo. S. Hort. 5:7-18.

Schnell RJ, J S Brown, C T Olano, E J Power, C A Krol, D N Kuhn, J C Motamayor (2003) Evaluation of avocado germplasm using microsatellite markers. J. Amer. Soc. Hort. Sci. 128:881-889.

Sharon D, J Hillel, S Mhameed, P Cregan, E Lahav, U Lavi (1998) Association between DNA markers and loci controlling avocado traits. J. Amer. Soc. Hort. Sci. 123:1016-1022.

Torres A M, B O Bergh (1980) Fruit and leaf isoenzymes as genetic markers in avocado. J. Amer. Soc. Hort. Sci. 116:10781081.

Virk P, H J Newburry, B V Ford-Lloyd (1998) AFLP reveals diversity in avocado (Persea americana Mill.). In: International Plant \& Animal Genome VI Conference (http://www.intlpag.org/6/abstracts/117.htm).

Vos P, R Hogers, M Bleeker, M Reijans, T van de Lee, M Hornes, A Frijters, J Pot, J Peleman, M Kuiper, M Zabeau (1995) AFLP: a new technique for DNA fingerprinting. Nucleic Acids Res. 23:4407-4414.

Yamamoto T, T Kimura, Y Sawamura, T Manabe, K Kotobuki, T Hayashi, Y Ban, N, Matsuta (2002) Simple sequence repeats for genetic analysis in pear. Euphytica 124:129-137. 